\title{
Artículos
}

\section{De reversa y llegando por primera vez a México. Rasgos sociodemográficos de niños y adolescentes que migran de Estados Unidos a México}

\section{In reverse and arriving in Mexico for the first time. Sociodemographic characteristics of children and adolescents migrating from the United States to Mexico}

\author{
Eduardo Carrillo Cantú* \\ Betsabé Román González**
}

\begin{abstract}
Resumen
Este artículo pretende ser el punto de partida para estudiar distintas agrupaciones de niños y adolescentes que migraron con sus familias de Estados Unidos a México, tomando en cuenta aspectos sociodemográficos y etnográficos relacionados con la llegada al país. Para 2010, en México se identificaron más de 570 mil menores de edad nacidos en Estados Unidos y más de 60 mil menores mexicanos retornados, por lo que su llegada representa un flujo migratorio inédito en la historia binacional. Estos niños y adolescentes conforman la llamada generación 0.5, y en sus trayectorias migratorias se encuentran experiencias subjetivas poco estudiadas.
\end{abstract}

Palabras clave: generación 0.5; migración de retorno; etnografía; demografía; menores migrantes internacionales.

\footnotetext{
Abstract

This article is intended as a starting point for studying various groups of children and adolescents who migrated with their families from the United States to Mexico,

* Instituto Tecnológico y de Estudios Superiores de Monterrey, Escuela de Ciencias Sociales y Gobierno. Dirección: Eugenio Garza Sada 2501 Sur, 64849, Monterrey, Nuevo León, México. Correo: eduardo.carrillo.cantu@gmail.com ORCID: https://orcid.org/0000-00033264-265X

** El Colegio de Sonora. Dirección: Obregón 54, Centro, 83000, Hermosillo, Sonora, México. Correo: broman@colson.edu.mx ORCID: https://orcid.org/0000-0001-9566-3533 
considering sociodemographic and ethnographic aspects related to their arrival in the country. In 2010, over 570000 children born in the United States and more than 60000 Mexican child returnees were identified in Mexico, representing an unprecedented migratory flow in binational history. These children and adolescents constitute generation 0.5 and their migratory trajectories include subjective experiences that have scarcely been studied.

Keywords: generation 0.5; return migration; ethnography; demography; international migrant children.

\section{Introducción}

La migración entre México y Estados Unidos ya no es como solía ser antes de 2007: la pérdida de más de ocho millones de empleos en Estados Unidos (Ramírez y Meza, 2011), el fortalecimiento en las políticas antiinmigrantes y el endurecimiento de la seguridad fronteriza entre ambos países, detonaron un cambio en el flujo migratorio (Escobar, Lowell y Martin, 2013). De acuerdo con Giorguli y Gutiérrez (2011), el Censo de Población y Vivienda 2010 reportó que en México se encontraban más de un millón de personas provenientes de Estados Unidos, y que gran parte de este contingente eran menores de edad de nacionalidad estadounidense.

Ahora bien, según datos de la Encuesta Nacional de la Dinámica Demográfica 2014 (Enadid), las familias migrantes señalaron que la principal causa de su retorno hacia México fue la reunificación familiar (61\%), seguido de otras razones como la deportación (16\%) y el cambio de trabajo (6\%) (Gonzalez-Barrera, 2015). Entre las personas que retornaron se identificó la presencia de más de 500 mil menores migrantes internacionales nacidos en Estados Unidos y más de 60 mil menores mexicanos con residencia previa en Estados Unidos (INEGI, 2017). No obstante, los menores migrantes se vuelven invisibles al pisar suelo mexicano, es decir, no hay un registro formal de su llegada ni de sus experiencias de vida en Estados Unidos. Esto representa un reto para ambos países porque los niños y adolescentes son agentes activos en la migración internacional y, generalmente, su bienestar y la unión familiar son las principales motivaciones que tienen las familias mexicanas para revertir la migración hacia México (Zúñiga, 2015).

Recientemente se han observado dos características en los estudios sobre migración entre México y Estados Unidos. En primer lugar, México se había estado estudiando como país expulsor de migrantes. Sin embargo, a partir del periodo de la gran crisis económica estadounidense (2007-2009), esta tendencia se revirtió haciendo al país un gran receptor no sólo de mi- 
grantes de retorno de nacionalidad mexicana, sino también de una significativa cantidad de menores nacidos en Estados Unidos (Passel, Cohn y Gonzalez-Barrera, 2012).

En segundo lugar, como bien señalaron Orellana, Thorne, Chee y Lam (2001), los estudios migratorios explicaban la experiencia migratoria de niños y adolescentes desde una perspectiva adultocéntrica, en la cual se considera que los menores son extensiones de sus padres ("maletas", como señalan las autoras), acompañando a los adultos en sus movimientos migratorios nacionales o internacionales (Dobson, 2009). Otros estudios explican que este adultocentrismo en los estudios con menores y en las investigaciones sobre migración invisibiliza, en general, las complejas experiencias cotidianas de niños y adolescentes en contextos escolares, familiares y sociales (Gaitán, 2006; Prout, 2011), y en particular, las destrezas metalingüísticas y académicas, las afiliaciones culturales y políticas, y los sentimientos de los menores migrantes internacionales en contextos transnacionales (Sánchez y Zúñiga, 2010; Hatfield, 2010; Panait y Zúñiga, 2016; Román, Carrillo y Hernández-León, 2016).

Es así que este artículo hace un acercamiento estadístico a las distintas agrupaciones de niños y adolescentes migrantes internacionales que salieron de Estados Unidos y se encuentran en México, partiendo de la información disponible de estos menores en las muestras estadísticas de los censos y las encuestas intercensales del INEGI; al tiempo en el que se presentan viñetas etnográficas en las que se dibujan trayectorias subjetivas de niños y adolescentes que se inscriben en lo que Víctor Zúñiga (Tecnológico de Monterrey), Silvia Giorguli (El Colegio de México), Edmund T. Hamann (Universidad de Nebraska en Lincoln), Juan Sánchez (Instituto de Investigación, Innovación y Estudios en Posgrado para la Educación), Betsabé Román (Universidad de California en San Diego), Eduardo Carrillo (Tecnológico de Monterrey) y otros investigadores han llamado la generación 0.5: aquellos menores de edad que experimentan una serie de rupturas, discontinuidades y negociaciones cotidianas producidas por el enfrentamiento de universos simbólicos rivales (escolares, culturales y políticos) en el contexto de su migración internacional.

Lo anterior se presenta con el fin de responder: ¿cómo son, sociodemográficamente, las características de la llegada a México de niños y adolescentes que migran de Estados Unidos?, ¿cuáles son las agrupaciones que pueden dibujarse, considerando las diferencias en sus trayectorias migratorias? Sus múltiples respuestas pueden servir como el punto de partida para que antropólogos, sociólogos, pedagogos y legisladores conozcan profundamente la heterogeneidad de las experiencias migratorias de los menores 
de edad que están llegando por primera vez a México, o que retornan después de vivir en Estados Unidos.

El texto está estructurado de la siguiente manera: en primer lugar, se presenta un panorama general de la estructura demográfica de la población menor de edad de origen mexicano y estadounidense (de padres mexicanos) en Estados Unidos, a partir del cambio del flujo migratorio internacional entre 2005 y 2010; luego se muestra cómo este viraje modificó la intensidad migratoria de retorno en México. En segunda instancia, se describe la metodología para la elaboración de este artículo, en el que, además de mencionar las fuentes estadísticas consultadas en ambos países, se presentan como antecedentes algunos de los resultados que se han obtenido de distintos estudios dirigidos por Víctor Zúñiga y que han sido la inspiración principal para constituir conceptualmente la generación 0.5 . En tercer lugar, se presenta una breve discusión teórica sobre la conformación de este concepto, que abre paso a la caracterización sociodemográfica de los cuatro grupos de menores migrantes internacionales de los que se tiene información. Por último, se abre una discusión sobre un quinto grupo, del cual, por su reciente identificación, se tiene poca información en ambos países: el de los niños y adolescentes que nacieron y vivieron en Estados Unidos, migraron hacia México y posteriormente han retornado a Estados Unidos y/o están circulando entre ambos países; todo esto antes de cumplir los 18 años.

\section{Estados Unidos como expulsor de migrantes}

La población mexicana en Estados Unidos había logrado conformarse por una comunidad de más de 11.5 millones de personas hacia 2015, de las cuales, alrededor de $54.9 \%$ eran niños y jóvenes de 0 a 20 años de edad ${ }^{1}$ (López y Radford, 2017). Quince años atrás, es decir, en el año 2000, el U.S. Census Bureau estimó que, entre 1995 y 2000, el país había recibido un flujo acumulado de inmigración mexicana de más de tres millones de personas -aproximadamente 600000 mexicanos anualmente-, y que, durante el mismo periodo, sólo 870000 mexicanos (incluidos los que nacieron en Estados Unidos) habían emigrado hacia México (Passel y Cohn, 2009). En la Gráfica 1 se muestra cómo la tradición expulsora de mexicanos hacia el norte, entre 2005 y 2010 , se revirtió con 1.39 millones de personas que migraron

${ }^{1}$ En esta cifra se incluye el grupo de 18 a 20 años, ya que la American Community Survey identifica grupos quinquenales de edad sin posibilidad de desglose del último grupo (de 16 a 20 años). 
de norte a sur, mientras que 1.37 millones de mexicanos emigraron a Estados Unidos; en síntesis, 1.57 millones de mexicanos menos que diez años antes (Passel, Cohn y Gonzalez-Barrera, 2012). Más adelante, entre 2010 y 2014, en un cambio histórico, la relación migratoria entre ambos países resultó negativa para Estados Unidos, con un déficit de 130 mil mexicanos emigrantes. En suma, desde 2005 el flujo migratorio tradicional de México a Estados Unidos se revirtió, transformando a México en un país receptor de migrantes internacionales, principalmente para menores de edad nacidos en Estados Unidos.

\section{Gráfica 1}

Número de mexicanos que inmigran y emigran en Estados Unidos, 1995-2014

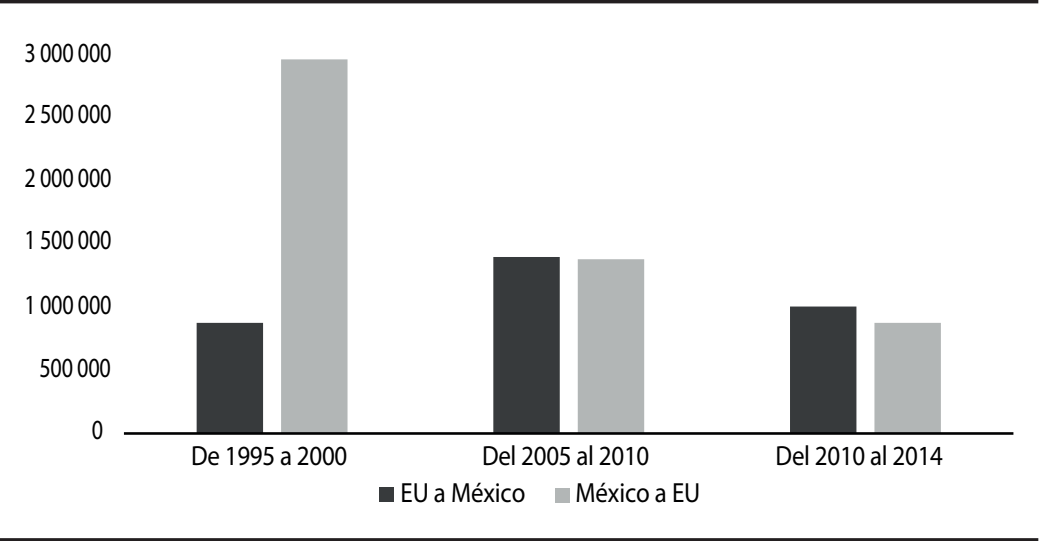

Fuente: Elaboración propia con información del American Community Census (U.S. Census Bureau, 2016) y de Passel, Cohn y Gonzalez-Barrera (2012).

Afortunadamente, tanto los censos estadounidenses como los mexicanos permiten encontrar subpoblaciones demográficas en estos flujos migratorios y distinguir el tamaño de distintos grupos de edad de niños y adolescentes mexicanos en Estados Unidos. Particularmente, el U.S. Census Bureau provee cohortes generacionales desde los 0 hasta los 20 años, ${ }^{2}$ en las que se muestra la cantidad de mexicanos que residen en el país, según grupos de edad. Si se contabilizan únicamente los niños y adolescentes de 0 a 15 años nacidos en México, se estima que 4.4 millones de menores esta-

${ }^{2}$ El U.S. Census Bureau no permite descomponer el grupo de edad de 16 a 20 años, por lo que aquí se muestra el grupo completo para incluir a los menores de 16 y 17 años de edad. 
ban residiendo en Estados Unidos en 2015. El principal grupo etario es el de 11 a 15 años de edad; es decir, aquellos que, muy probablemente, cursaron algunos años de escuela en Estados Unidos. El siguiente grupo, el de mexicanos de 6 a 10 años de edad, representa el segundo contingente de mayor relevancia estadística. Por último, el grupo de menores que no se encuentran en edad escolar representa una población cercana al millón de personas que nacieron en México, pero que muy probablemente iniciarán sus estudios escolares en Estados Unidos, algunos de ellos retornarán a México con sus familias, y otros tendrán estancias más prolongadas en Estados Unidos (U.S. Census Bureau, 2016).

\section{Gráfica 2}

Población nacida en México y que reside en Estados Unidos 2015, según grupos de edad

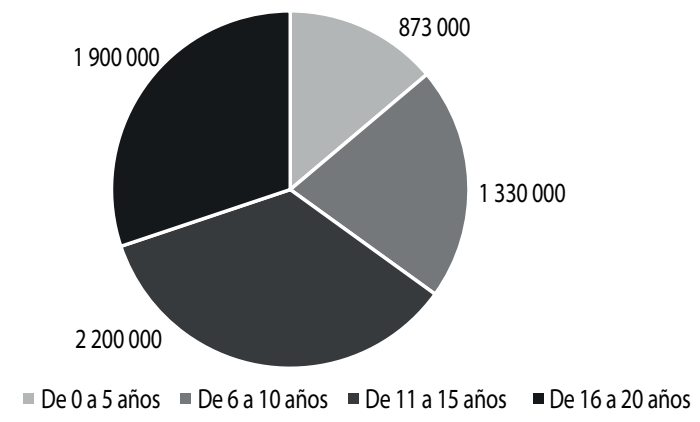

Fuente: Elaboración propia con información del American Community Census (U.S. Census Bureau, 2016).

\section{Niños y adolescentes mexicanos en cuatro entidades de Estados Unidos}

Si bien la dispersión geográfica de los migrantes mexicanos en Estados Unidos se encuentra cada vez más extendida en destinos de menor tradición migratoria (Zúñiga y Hernández-León, 2005; Passel y Cohn, 2016; Stepler y Lopez, 2016), California, Texas, Illinois y Arizona siguen concentrando la mayor parte de esta población migrante. Mientras que los primeros dos estados albergan la mayoría de los mexicanos migrantes -4.2 millones y 2.54 millones, respectivamente-, Illinois y Arizona han mostrado cambios 
significativos en la cantidad de mexicanos, especialmente para aquellos de 0 a 17 años de edad.

En el Cuadro 1 se muestra la cantidad total de población mexicana en los cuatro estados mencionados, así como el porcentaje de los grupos etarios de 0 a 5 años y de 5 a 17 años, y sus cambios en 2010 y 2016. El primer grupo muestra una ligera disminución porcentual en todos los estados, lo cual puede significar que, para estos estados, la intensidad de la inmigración se ha mantenido constante o ha disminuido muy poco. Sin embargo, el segundo grupo, aquel que corresponde a los niños y adolescentes en edad escolar, disminuyó en California, Texas e Illinois casi 50\% entre ambas muestras. Por el contrario, Arizona es el que muestra un incremento significativo, de $7.7 \%$ en 2010 a $22.3 \%$ en 2016 , lo que significa que tiene una población mexicana mucho más joven que en los años anteriores por tres posibles razones: emigraron más adultos que niños y adolescentes, inmigraron al estado más menores de este grupo de edad, o una combinación de las dos primeras.

\section{Cuadro 1}

Porcentaje de población de 0 a 5 años y de 5 a 17 años nacida en México de la población total de mexicanos en cada estado de Estados Unidos, según grupo de edad; 2010 y 2016

\begin{tabular}{lccccccc}
\hline & \multicolumn{4}{c}{$\mathbf{2 0 1 0}$} & & \multicolumn{3}{c}{$\mathbf{2 0 1 6}$} \\
\cline { 2 - 3 } \cline { 7 - 8 } Estado & $\begin{array}{c}\text { \% 0-5 } \\
\text { años }\end{array}$ & $\begin{array}{c}\text { \% 5-17 } \\
\text { años }\end{array}$ & $\begin{array}{c}\text { Total } \\
\text { población }\end{array}$ & $\begin{array}{c}\text { \% 0-5 } \\
\text { años }\end{array}$ & $\begin{array}{c}\text { \% 5-17 } \\
\text { años }\end{array}$ & $\begin{array}{c}\text { Total } \\
\text { población }\end{array}$ \\
\hline California & 0.4 & 6.2 & 4314580 & & 0.3 & 3.3 & 4235643 \\
Texas & 0.5 & 8.1 & 2485336 & & 0.5 & 4.6 & 2589058 \\
Illinois & 0.5 & 6.1 & 708580 & & 0.3 & 3.0 & 653367 \\
Arizona & 0.5 & 7.7 & 517443 & & 0.4 & 22.3 & 509931 \\
\hline
\end{tabular}

Fuente: Elaboración propia con información del American Community Census (U.S. Census Bureau, 2010; 2016).

Para estos mismos estados, el tiempo de residencia del total de su población mexicana ha seguido las mismas tendencias nacionales de llegada y asentamiento en Estados Unidos. Para el año 2010, California era el estado con la población mexicana más antigua que el resto de las entidades mencionadas, pero mostraba un ligero incremento porcentual entre sus emigrantes más recientes (de 2000 en adelante) y los de mayor antigüedad (entre los 10 y 20 años). En comparación, Texas se presentó como 
el estado con emigrantes más recientes en su llegada a Estados Unidos. Sin embargo, el panorama para 2016 evidencia un cambio rotundo en los asentamientos mexicanos en estos cuatro estados. California se mantiene como la entidad con una población más antigua, pero con uno de los flujos de llegada menos grandes. El caso de Arizona sobresale del resto porque, a diferencia de hace seis años, muestra asentamientos más antiguos que los que reportó en el censo de 2010 (U.S. Census Bureau, 2010; 2016). En corto, estos cambios en el asentamiento de la población mexicana en los estados de mayor recepción migrante revelan no sólo lo que Zúñiga y Hernández-León (2005) denominaron como "los nuevos destinos de la migración" (una dispersión geográfica más extendida y diversa en Estados Unidos), sino también supone una serie de cambios en la estructura demográfica de esas entidades.

\section{Cuadro 2}

Porcentaje de la población nacida en México

en cada estado de Estados Unidos, según año de llegada al país y total de la población mexicana; 2010 y 2016

\begin{tabular}{|c|c|c|c|c|c|c|}
\hline \multirow[b]{2}{*}{ Estado } & \multicolumn{3}{|c|}{2010} & \multicolumn{3}{|c|}{2016} \\
\hline & $\begin{array}{l}2000 \text { en } \\
\text { adelante }\end{array}$ & $\begin{array}{c}1990- \\
1999 \\
\end{array}$ & $\begin{array}{c}\text { Antes } \\
\text { de } 1990\end{array}$ & $\begin{array}{l}2010 \text { en } \\
\text { adelante }\end{array}$ & $\begin{array}{c}2000- \\
2009\end{array}$ & $\begin{array}{c}\text { Antes } \\
\text { de } 2000\end{array}$ \\
\hline California & 24.7 & 23.8 & 23.5 & 5.5 & 21.3 & 39.8 \\
\hline Texas & 31.5 & 26.1 & 19.2 & 11.0 & 24.9 & 35.7 \\
\hline Illinois & 27.9 & 30.1 & 16.7 & 5.1 & 22.5 & 39.0 \\
\hline Arizona & 28.4 & 27.0 & 19.1 & 7.7 & 22.3 & 38.0 \\
\hline
\end{tabular}

Nota: El porcentaje faltante de la suma de los tres periodos de llegada por cada año y estado corresponden a periodos de mayor antigüedad y no se presentan desglosados en el U.S. Census Bureau.

Fuente: Elaboración propia con información del American Community Census (U.S. Census Bureau, 2010; 2016).

\section{El cambio en la intensidad de retorno en México}

Otro de los grandes cambios demográficos que proyecta el retorno, es el de la intensidad migratoria en las entidades federativas que reciben a los migrantes en México. El Consejo Nacional de Población (Conapo) proporciona, en el índice de intensidad migratoria, el porcentaje de viviendas con migrantes que retornaron dentro de los cinco años anteriores al censo. Este 
recurso estadístico provee un panorama nacional, regional y municipal de las geografías de retorno que se han extendido desde el año 2000. Este material, como se verá en el siguiente apartado, muestra aquellos territorios que han reportado un cambio en su flujo de recepción y que son uno de los puntos de partida más importantes para el estudio de la migración de retorno de niños y adolescentes mexicanos, así como de los hijos nacidos en Estados Unidos de padres y madres mexicanos.

Este índice muestra una composición más completa de las viviendas mexicanas que están expuestas a la migración internacional y divide el territorio mexicano en cuatro regiones: Tradicional, Norte, Centro y Sur-sureste. $^{3} \mathrm{Si}$ bien este producto no alcanza a perfilar la población menor de edad, sí permite generar un panorama amplio de la situación mexicana. En la Gráfica 3 se muestra el porcentaje de viviendas con migrantes de retorno por cada estado, los cuales se encuentran ordenados de forma descendente, tomando en cuenta el porcentaje de viviendas con migrantes de retorno en el año 2010. Para el último índice, el porcentaje nacional de viviendas con este tipo de migrantes fue de $2.2 \%$, muy superior al $0.9 \%$ registrado en 2000 . Asimismo, todas las entidades del país reflejaron un incremento, en términos absolutos, mucho mayor en el año 2000. Las cinco entidades con mayor porcentaje de viviendas pertenecen a la región Tradicional: Zacatecas (de 2.6 a 5.6\%), Michoacán (de 2.4 a 4.8\%), Guanajuato (de 1.7 a 4.1\%), Nayarit y Colima (ambas de 2.1 a 4\%); y dos estados correspondientes a la región Centro: Hidalgo (de 0.9 a 4\%) y Morelos (de 1.2 a 3.5\%) (Conapo, 2011).

Sin embargo, en términos de crecimiento porcentual del año 2000 al 2010, Hidalgo y Oaxaca (de 0.7 a 3.1\%) encabezan el incremento porcentual y reflejan, indudablemente, la extensa dispersión geográfica del retorno en el país. Los más de un millón de personas que salieron de Estados Unidos a México, reportados por el U.S. Census Bureau, llegaron a todos los estados de México, pero con diferencias notables entre regiones y microrregiones (Zúñiga, 2015), haciendo de este fenómeno un flujo heterogéneo y compuesto por muchos subgrupos poblacionales, entre ellos, los niños y adolescentes mexicanos y aquellos que nacieron en Estados Unidos.

${ }^{3}$ El Conapo identifica a la región Tradicional con los siguientes estados: Aguascalientes, Colima, Durango, Guanajuato, Jalisco, Michoacán, Nayarit, San Luis Potosí y Zacatecas. La región Norte se compone por: Baja California, Coahuila, Chihuahua, Nuevo León, Sonora, Tamaulipas, Baja California Sur y Sinaloa. La región Centro incluye las entidades de: Morelos, Querétaro, Tlaxcala, Puebla, Hidalgo, Distrito Federal (hoy Ciudad de México) y Estado de México. Finalmente, en la región Sur-sureste se encuentran: Campeche, Chiapas, Guerrero, Oaxaca, Quintana Roo, Tabasco, Veracruz y Yucatán. 


\section{Gráfica 3}

Porcentaje de viviendas con migrantes

de retorno en cada estado de México, 2000 y 2010

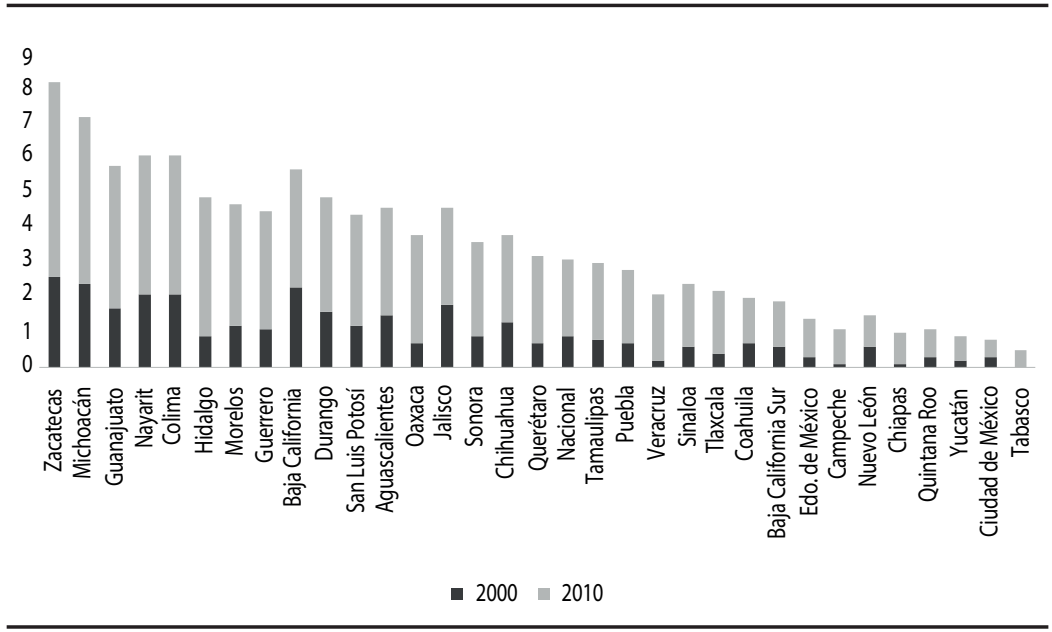

Nota: El diseño de la gráfica muestra el total de los porcentajes de viviendas con migrantes de retorno para cada estado; se encuentra ordenada de manera decreciente, tomando en cuenta el valor porcentual del año 2010.

Fuente: Conapo, 2011.

\section{Metodología}

Como se precisó con anterioridad, los datos censales que ofrece el American Community Census y el INEGI son el punto de partida para trazar los nuevos flujos migratorios en los cuales se inscribe la población menor de edad que ha nacido en cada uno de los dos países. Si bien los datos estadísticos ofrecen un panorama general de las características sociodemográficas que caracterizan a los niños y adolescentes migrantes internacionales, hay una serie de limitaciones metodológicas que no permiten conocer las experiencias familiares, escolares, culturales y migratorias de los subgrupos que se encuentran en tránsito entre ambos países. Por ejemplo, los censos en ambos países únicamente captan a los menores que se encontraban en el país (México o Estados Unidos) hasta cinco años antes del levantamiento de la información; aquellos de mayor antigüedad, o los que llegaron al país después de la aplicación de la encuesta, no son reflejados en las muestras estadísticas. No obstante, esta limitación también se presenta de forma inversa, ya que los menores que abandonaron el país antes del censo no son 
capturados numéricamente, ocultando la cantidad de migrantes circulares que podría reflejar cada censo dentro del lustro en el que se contempla el periodo de retorno (Zúñiga, 2015; Román, 2017). Dicho en otras palabras, las bases de datos señalan que existe una población que permanece invisible en las escuelas y comunidades mexicanas.

Para fines de este artículo, se combinan dos principales fuentes de información que ayudan a dibujar los cinco perfiles de niños y adolescentes migrantes internacionales que se plantean como hallazgos. En primer lugar, se consultaron los tabulados básicos y las consultas interactivas en la serie histórica 1990-2010 de los Censos y Conteos de Población y Vivienda del INEGI y la Encuesta Intercensal 2015 para la caracterización por nacionalidad (nacidos en México y en Estados Unidos), grupos de edad, tamaño de residencia y corresidencia compartida 2000 y 2010. En segundo lugar, se muestran los hallazgos de cinco estudios que se han realizado en escuelas mexicanas y que han permitido: 1) identificar las agrupaciones de los niños y adolescentes migrantes, a partir del trabajo de campo por más de trece años; 2) trazar las directrices para profundizar en las diferencias demográficas que tienen unas agrupaciones de otras; y 3) retratar etnográficamente los sentimientos, expectativas, narrativas y deseos de los menores.

\section{Cinco casos de estudio en escuelas mexicanas}

Desde 2004, Víctor Zúñiga, Juan Sánchez, Edmund T. Hamann y un equipo de investigadores de la Universidad de Monterrey (UDEM), del Centro de Investigaciones y Estudios Superiores en Antropología Social (CIESAS) y del Tecnológico de Monterrey, realizaron el levantamiento de cinco muestras estatales en distintas entidades de México para examinar el fenómeno de la migración de retorno de familias migrantes mexicanas y para establecer estimaciones locales y nacionales sobre la cantidad de alumnos transnacionales en México, así como mostrar las diferencias regionales de este fenómeno en el país (Zúñiga, Hamann y Sánchez, 2008).

Por medio de las primeras cuatro muestras representativas -Nuevo León, 2004; Zacatecas, 2005; Puebla, 2009; y Jalisco, 2010-, se encuestaron a 56100 niños y adolescentes entre los siete y dieciséis años de edad, ${ }^{4}$

${ }^{4}$ El procedimiento metodológico para las cuatro muestras se realizó en forma representativa de todo el universo escolar de educación básica de cada estado. Los alumnos de los primeros tres grados escolares fueron encuestados oralmente, mientras que a los de cuarto a sexto de primaria y los tres grados de secundaria se les encuestó de manera escrita. Para la selección de los salones se realizó un muestreo aleatorio de cada grado, en los cuales se les entregó un 
inscritos en escuelas primarias y secundarias de dichos países. A partir de estos estudios, el equipo de investigación pudo trazar estadística y etnográficamente un bosquejo de las tipologías de los menores migrantes internacionales en México. De estas muestras, Sánchez y Zúñiga (2010) y Zúñiga y Hamann (2014) estimaron que para el 2010, se encontraban más de 420000 alumnos transnacionales con experiencia escolar previa en Estados Unidos, y que en ese momento se encontraban en escuelas mexicanas, divididos en dos grupos: alumnos binacionales (nacidos en Estados Unidos) y alumnos mononacionales (nacidos en México). Sin embargo, aun cuando las entrevistas con 179 alumnos transnacionales proveyeron información valiosa sobre las experiencias de retorno de estos menores, se pasaban por alto otras experiencias cotidianas que sólo es posible capturar por medio del trabajo etnográfico (Hamann, Zúñiga y Sánchez, 2006; Zúñiga, 2013).

Considerando las limitaciones anteriores, Betsabé Román, junto con un equipo interdisciplinario, desarrolló un estudio longitudinal entre 2012 y 2015 en el estado de Morelos con el objetivo particular de narrar las complejidades en las trayectorias migratorias de los niños y adolescentes que conforman estas tipologías. La selección de este estado estuvo determinada por dos criterios: 1) Morelos es de las pocas entidades en México en la cual el Probem (Programa Binacional de Educación Migrante) posee un registro estatal de la llegada de menores de edad de Estados Unidos que se inscribieron en escuelas de educación básica en el estado: esta base de datos incluye el año de llegada, los años cursados en Estados Unidos, el lugar de proveniencia, la nacionalidad del alumno, la escuela y el grado en el que se le inscribió al llegar a la escuela morelense, y por último, los datos de contacto; y 2) este estado fue identificado por el índice de intensidad migratoria como una entidad de alta intensidad migratoria de retorno.

Se realizó una muestra por conveniencia del universo escolar de escuelas públicas y privadas de educación básica para identificar aquellas primarias y secundarias que mostraran una alta presencia de alumnos transnacionales en alguno de sus grados escolares. Al final, se encuestaron 1395 alumnos de cuarto a sexto de primaria y los tres grados de secundaria, con un rango de edad de ocho a dieciséis años; de ellos, 87 eran alumnos con experiencia escolar previa en Estados Unidos (de este grupo, 62 eran de nacionalidad mexicana, mientras que 23 eran binacionales). Posteriormente,

cuestionario general a todos los alumnos, más un cuestionario específico para los no migrantes, y otro para los transnacionales. Algunos de los estudiantes de este último grupo compartieron sus experiencias por medio de una entrevista. 
Román y Carrillo le dieron seguimiento por tres años y medio a 10 niños y adolescentes (cinco hombres y cinco mujeres) en los municipios morelenses de Cuernavaca, Jiutepec, Tlaltizapán, Jojutla, Cuautla y Temixco, y en una localidad del municipio de Ocuilan, en el Estado de México (Román y Carrillo, 2017; Román, 2017). Las siguientes secciones están dedicadas especialmente a niñas, niños y adolescentes como Lulú, Elena, Beto, Marco y Flor, que desdibujan las conceptualizaciones con las que se ha mirado a la infancia migrante y enriquecen la comprensión de la historia de vida de los menores que experimentan la migración internacional de formas poco observadas.

Para lograr lo anterior, se analizan las fuentes estadísticas disponibles en México que describen sociodemográficamente a la población total de 0 a 17 años de edad, el grupo migrante de 5 a 17 años (no se incluyen personas de 0 a 4 años porque no habían nacido en el momento del censo; esto se explicará más adelante), y el grupo en edades escolares de 6 a 17 años, según su nacionalidad y otras variables relacionadas a la llegada al país. Ahora bien, sólo fue posible distinguir estas características específicas de las cohortes que componen el grupo de población de los menores de edad por el trabajo etnográfico que ha sido realizado con alumnos transnacionales en escuelas mexicanas (Zúñiga, Hamann y Sánchez, 2008) y por el estudio longitudinal realizado en el estado de Morelos (Román, 2017).

\section{Los niños y adolescentes de la generación 0.5}

A diferencia de otras generaciones conceptualizadas en los estudios migratorios, como la generación 1.5 o la segunda generación, los niños y adolescentes que ahora se encuentran en México experimentan procesos de socialización con otros tipos de fragmentación y complejidad que los jóvenes que se inscriben en las anteriores generaciones. Ya Rumbaut (2004) advertía, desde la década de los noventa, sobre las diferencias lingüísticas y de aculturación que experimentan los menores migrantes en distintas edades. Esto es, que aun cuando la población menor de edad representa un conjunto demográfico delimitado por la legalidad (antes de cumplir la mayoría de edad), sus diferencias culturales y sociales pueden dividirse por cohortes en las que los niños y adolescentes experimentan distintos tipos y grados de aculturación en las comunidades receptoras: lo que él llamará la infancia temprana (de 0 a 5 años), la infancia media (de 6 a 12 años) y la adolescencia (13 años o más), y que determinarán, en gran medida, la influencia de la socialización familiar y escolar de los menores migrantes e hijos de 
migrantes de primera generación en su futuro y subjetividad en el país de destino de sus padres.

Las cohortes de Rubén Rumbaut dirigen la mirada hacia las características familiares, sociales, culturales, lingüísticas, étnicas y socioeconómicas que vinculan a los niños y adolescentes con sus padres migrantes. $\mathrm{Su}$ estudio, sin embargo, se realiza considerando un asentamiento prolongado de estos menores (hasta llegar a la adultez), por lo que, en su proceso de socialización escolar, no se encontrarán tantas contradicciones y malentendidos generados por la migración internacional (Román y Zúñiga, 2014): si un adolescente mexicano migra hacia Estados Unidos es más probable que mantenga un vínculo semántico, normativo y emocional hacia su comunidad de origen, por lo que en Estados Unidos tenderá a parecerse más a los jóvenes de la generación 1.5; pero si un hijo de migrantes mexicanos crece en Estados Unidos de manera prolongada, la socialización escolar, es decir, la transmisión de códigos, normas y valores estadounidenses, terminará por imponerse a la socialización familiar, lo que lo conformaría en un joven de la segunda generación (Rumbaut, 1997; Zhou, 1997, Portes y Hao, 2004; Gonzales, 2010). Por estas razones, los cientos de miles de niños y adolescentes que iniciaron o continuaron su escolaridad en Estados Unidos y que ahora se encuentran en México, se alejan de estas categorías conceptuales (generación 1.25, 1.5, 1.75 o segunda generación) porque la migración internacional y la inscripción en un sistema escolar implica una fragmentación en el proceso de socialización de estos menores (Zúñiga, 2011; Zúñiga y Hamann, 2011; Zúñiga y Vivas, 2014; Román y Carrillo, 2017).

Las teorías de socialización, como la de Berger y Luckmann (2003), indican que es durante la infancia en donde se desarrolla la internalización del mundo social en el cual se desenvolverán los niños y adolescentes durante toda su vida. Se presenta sin mayores alteraciones, ya que, por lo general, la escuela es la primera institución encargada de transmitir imaginarios, normas, códigos, símbolos y formas de interpretar la realidad (Durkheim, 1976; Dubet, 2002). Es una socialización contundente. Sin embargo, los menores que llegan a México, y que tienen iniciada una socialización escolar en un sistema educativo de otro país, se insertan en un nuevo mundo social, escolar, lingüístico y simbólico que rivaliza con el mundo internalizado anteriormente, en plena socialización primaria.

Por esta razón, Zúñiga y Giorguli (2019) otorgan una nomenclatura especial a esta población, identificada como la generación 0.5 , que se constituye por: 
[...] componentes objetivos, como son el bilingüismo de los niños, su conocimiento práctico de las dos sociedades en donde se educaron, los conocimientos académicos adquiridos (historia, geografía de ambos países) y las destrezas para negociar con múltiples contextos. Y contiene también componentes subjetivos, como son los sentimientos de pertenencia, el apego a ciertos símbolos, afiliaciones múltiples y lealtades [...] Niños y adolescentes de la segunda generación o de la generación 1.5 se convirtieron en niños de la generación 0.5 : son migrantes internacionales viviendo y educándose en México y son, a la vez, mexicanos criados en Estados Unidos y, algunos, estadounidenses educándose en México.

\section{Definiendo a los distintos grupos de niños, niñas y adolescentes de la generación 0.5}

Los siguientes extractos pertenecen a las historias de vida de menores migrantes que integran la generación 0.5 ; ellos son parte de seis familias en Morelos, y ponen en relieve distintas trayectorias migratorias. Algunas características generales de las familias que se incluyeron son: $a$ ) en todas las familias, ambos padres eran de origen mexicano; b) todas las familias expresaron que hubo algún lapso de separación entre padres e hijos, ya sea en Estados Unidos o en México; c) Beto y Marco, junto con sus padres, son retornados, mientras que Lulú, María y Flor llegaron a México por primera vez; d) pese al gran número de deportaciones anuales de Estados Unidos a México, solamente dos familias retornaron a México por deportación del padre de familia, mientras que las otras cuatro regresaron por otras causas que incluyeron: tener que cuidar a algún familiar que estaba enfermo en México; que los menores pudieran conocer a sus familias en México, así como su cultura y lenguaje; haber sido víctimas de extorsión en Estados Unidos, y/o miedo a la deportación del padre o la madre de familia. Siguiendo el ejemplo de Valdés (1996), se agruparon las familias de la siguiente manera: $i$ ) familias con hijos o hijas mexicanos y que son retornados en México (menores retornados); ii) familias con hijos o hijas nacidos en Estados Unidos que residen aún en México (menores binacionales en México); y iii) familias con hijos o hijas americanos y que ya han retornado a Estados Unidos (menores binacionales retornados a Estados Unidos) (Román, 2017).

Las agrupaciones que se definen más adelante poseen una caracterización predominantemente estadística en torno a sexo, división por grupos de edad, nacionalidad, escolaridad y lugares en México de mayor llegada. 


\title{
i) Menores retornados
}

\author{
Beto: mexicano que inició la escuela \\ en Estados Unidos y ahora está en México
}

Betito es el primer niño que conocí. Vive en Ahuatenco, Estado de México (a media hora en taxi de Cuernavaca), con sus papás en una casa que estaban construyendo mientras vivían en Estados Unidos. Beto nació en Cuentepec, en el árido municipio de Temixco, en Morelos. Ahí todavía viven su abuelita materna, su tía (media hermana de su mamá, que resulta ser un año menor que él) y el mayor de sus tíos; todos ellos hablan náhuatl porque Cuentepec sigue siendo una comunidad náhuatl. Beto se fue a Estados Unidos con su mamá cuando tenía dos meses: cruzó la frontera en Tijuana y se encontraron con su papá que ya tenía unos meses viviendo en Santa Ana, California. Betsabé Román y Víctor Zúñiga ya lo habían conocido en alguna reunión que tuvieron en la Secretaría de Educación Pública durante la graduación de la escuela secundaria de Cuentepec. Después de terminar el tercer grado (por error, porque en su escuela lo adelantaron al no saber cómo interpretar las calificaciones de Estados Unidos), se fue a vivir a Ahuatenco, un pueblito en una de las pendientes más elevadas de una montaña en el eje volcánico entre Morelos y el Estado de México. Beto está en la preparatoria de Cuernavaca, una privada en la que le enseñan cultura para la paz y globalización... En esta escuela habla inglés con su maestra de idiomas, pero le da pena ayudar a sus compañeros cuando su maestra lo invita a ser tutor de la materia. Luego de algunos semestres, tuvo que dejar esa preparatoria y se metió en una nueva que abrieron en Ahuatenco; el problema fue que perdió dos semestres porque en su escuela anterior no le quisieron entregar sus documentos oficiales. Ahora (2016) se acaba de reinscribir en la preparatoria de su comunidad y está a un par de semestres de terminar los estudios. Como cuando lo conocí (en 2014), sigue pensando en estudiar la universidad en Estados Unidos o irse a trabajar para mandarle dinero a sus papás, pero sabe que, como no nació allá, no tiene pasaporte (notas de campo de Eduardo Carrillo, 2014 y 2016).

Marco: mexicano que inició la escuela en México, la continuó en Estados Unidos y ahora está de regreso

Marco fue de los últimos niños que conocí en 2014. Habíamos intentado localizarlo todas las veces que íbamos a Morelos, pero su mamá no nos con- 
testaba, cancelaba nuestros encuentros o tenía problemas que atender con las dos hermanas mayores de Marco. Él es el único niño de los que acompañamos que inició la escuela en México, y después de estudiar tres años en Chicago, regresó a México con su mamá. Su papá tiene documentos, así que puede trabajar en Illinois sin problemas. La razón del regreso fue para estar juntos en México porque cuando migraron Marco (que tenía seis años) y su mamá, ésta dejó a sus dos hijas bajo el cuidado de la abuela de Marco, en Cuernavaca. Cuando llegaron a México, la mamá lo inscribió en una escuela privada para seguir practicando el inglés, para cuando regresara a Estados Unidos, pero le hicieron repetir el tercer grado de primaria (ya había estudiado ese grado en Chicago). Luego, lo cambiaron a una escuela primaria que queda más cerca de su casa y está enfrente de la cremería que tiene su mamá. Ahí también le hicieron repetir año (tercer grado) porque el director quería que tuviera "el mismo nivel" que sus compañeros... Domina muy bien el español escrito y leído y tiene calificaciones superiores a ocho. Quiere estudiar diseño gráfico, animación de videojuegos o arte y caligrafía. Nos dijo que se siente cómodo en México, no extraña mucho su vida en Estados Unidos, pero no tendría problema en volver allá (notas de campo de Eduardo Carrillo, 2014 y 2016).

\section{ii) Menores binacionales en México}

Lulú: estadounidense que inició la escuela en Estados Unidos y ahora está en México

Cuando conocimos a Lulú, en 2012, tenía 13 años y vivía con su tres hermanas y sus papás en Jojutla, Morelos. Se había mudado de Chicatlán, en el municipio de Tlalquitenango, y había llegado a Morelos un año antes. Lulú nació en Los Ángeles, California, pero desde pequeña migró con su papá, su mamá y su hermana mayor a Nebraska; después se asentaron en San Diego con su abuelita, su tía y sus primos, al norte de la ciudad. Sus papás no tienen documentos para vivir en Estados Unidos, pero sus cuatro hijas nacieron en aquel país y ahí vivieron hasta 2012, cuando Lulú y sus hermanas vieron cómo era deportado un tío. La familia se reunió para platicar sobre la posibilidad de retornar a México todos juntos o de separarse entre México y Estados Unidos. Después de escuchar la preocupación de sus padres, las cuatro niñas concordaron que lo mejor era regresar a México para mantener a su familia unida: "Yes, we are American, and yes, we could have stayed, but that would have meant to be away from our parents, that was not an op- 
tion for us. We were together there, we had to be together in Mexico too". Un año después de llegar a México con sus padres, Lulú nos comentó: "I feel as Mexican as cualquiera de los que están aquí. I have sangre mexicana in my blood". En ese momento nos enteramos que había decidido dejar el primer grado de secundaria porque una de sus maestras no sabía cómo enseñarles a alumnos migrantes internacionales. Pasaron dos ciclos escolares (entre 2014 y 2016) para que Lulú volviera a la secundaria, pero ahora (agosto de 2017) ya está en la preparatoria. Aun cuando ahora tiene 17 años, sigue pensando en estudiar una carrera en México para irse de vuelta a Estados Unidos y enviarle dinero a sus papás cuando trabaje allá, o en irse a estudiar al college o a la universidad en Estados Unidos (notas de campo de Eduardo Carrillo, 2014, 2015 y 2017).

\section{María: estadounidense que inició la escuela en México}

Ella es muy inteligente, aun cuando tiene cuatro años, entiende perfectamente el inglés cuando sus hermanas hablan entre ellas en ese idioma. María llegó en 2012 con Lulú -otra de las niñas que acompañamos-, sus otras dos hermanas y sus papás. Es la menor de la familia y nació en San Diego. Cuando hablo con ella en inglés me responde en español. Su mamá nos dice que María puede ver puras caricaturas y películas en inglés y les dice a sus hermanas mayores que le hablen en inglés para que no pierda el idioma; muy de vez en cuando, sí nos habla en inglés... Ella no estudió en Estados Unidos porque no había cumplido la edad para entrar a la escuela, pero comenzó a estudiar el jardín de niños en Morelos y casi no tiene recuerdos de su vida en San Diego (notas de campo de Eduardo Carrillo, 2014 y 2016).

\section{iii) Menores binacionales de retorno a Estados Unidos}

Flor: estadounidense que inició la escuela en Estados Unidos, la continuó en México y ahora está en Estados Unidos

Yo no he podido conocer personalmente a Flor: ella se regresó a Oregón con su papá, antes de que pudiera verla. Conozco bien a su mamá y siempre que vamos a Morelos le marcamos para platicar con ella y con sus hermanas mayores, y jugar con su sobrinito. Flor nació en Oregón, mientras su papá y su mamá seguían casados, pero después de una separación conflictiva y de los deseos de su mamá de reencontrarse con su hija mayor en Jiu- 
tepec, Morelos, llegó por primera vez a México. En Jiutepec estudió parte del primer año de secundaria, pero después de estar sólo cuatro meses, se quiso regresar a Estados Unidos. Su mamá nos dice que no aguantó que sus maestros en México no se dieran cuenta de que ella es estadounidense y por eso no sabe leer y escribir bien en español, no conoce historia ni geografía de México... Flor intenta viajar durante los veranos, cada vez que puede, para estar con su mamá en Morelos, pero como ella se mudó a la Ciudad de México para arreglar unos problemas legales que tiene por uno de los hijos de su hija mayor, no hemos podido hablar con ninguna de las dos para saber cómo le está yendo a Flor en Estados Unidos (notas de campo de Eduardo Carrillo, 2014 y 2015).

\section{Muchos estadounidenses iniciarán su escolaridad en México}

Como bien señaló Rubén Rumbaut, la edad importa mucho en el momento de migrar internacionalmente; además, la población de más de 570 mil menores de edad de nacionalidad estadounidense se compone principalmente por un contingente que se ubica en los primeros años de la primera infancia. En la Gráfica 4 se observa un comparativo en la población de 0 a 17 años nacida en Estados Unidos que llegó al país en 2000 y 2010. Para este último año, el grupo de menores con edad escolar de primero a cuarto grado de primaria constituye el bloque más extenso, con más de 208 mil niñas y niños; muy cerca de este grupo está el de los pequeños que no se encuentran en edad escolar; el tercer grupo, con casi 114 mil personas, es el de los de 10 a 14 años; y finalmente, en menor cantidad, el grupo de 15 a 17 años.

Ahora bien, el Conapo registró que los principales cinco estados receptores de migrantes internacionales se encontraban en la región Tradicional; sin embargo, el lugar de origen de sus padres no corresponde a tales estados ni a dicha región. Tal como se muestra en el Cuadro 4, la dispersión geográfica de los menores nacidos en Estados Unidos se incrementa conforme pasan los años. Así, en 25 años, la región Norte se mantiene como la principal región receptora para 2015, pero disminuye en cada año registrado, y aun cuando el porcentaje de origen es menor para las regiones Centro y Sur-sureste, el incremento, con respecto a las décadas anteriores, es notable porque estudios previos han identificado que los lugares de destino de retorno no siempre son los mismos lugares de origen de estos migrantes (Zúñiga y Hernández-León, 2005). De igual manera, la dispersión no sólo se manifiesta en las regiones delimitadas geopolíticamente, sino también en el crecimiento de las poblaciones que representan la ruralidad o la urbanidad de los luga- 
res de residencia de los menores migrantes internacionales. La tendencia es contundente: incluso cuando el lugar de residencia es menor a los 2500 habitantes para el $25.1 \%$ de los estadounidenses entre los 6 y 17 años de edad, estos niños y adolescentes están llegando cada vez más a localidades rurales.

\section{Gráfica 4}

Población de 0 a 17 años nacida en Estados Unidos

y que vive en México, según grupos de edad, 2000 y 2010

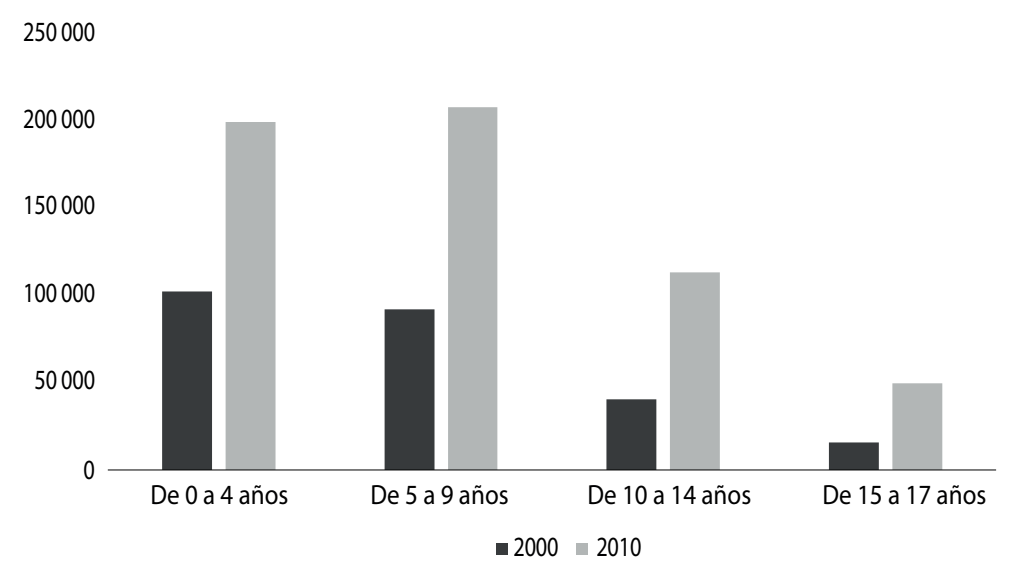

Fuente: Elaboración propia con información del Censo de Población y Vivienda INEGI, 2000 y 2010 (INEGI, 2017).

También la corresidencia del hogar revela algunas de las composiciones familiares en las que se configuran las familias mexicanas con hijos nacidos en Estados Unidos. Por ejemplo, para la población entre 6 y 17 años es más común encontrar a estos menores migrantes residiendo con ambos padres, pero cuando viven con uno de los dos, el porcentaje de menores que viven únicamente con la madre es muy superior en todos los grupos de edad. Como en el caso de Flor, aun cuando estuvo viviendo unos meses en México, ella compartió su residencia con su madre, mientras que su padre siguió viviendo en Estados Unidos. Esto es, para los nacidos en Estados Unidos, si tienen una red familiar robusta y extendida entre ambos países, la movilidad transnacional a futuro es mucho más sencilla de realizar que para los menores nacidos en México. Sin embargo, el caso de Flor es singular porque ella vivió con su madre en México y luego retornó a Estados Unidos para vivir con su padre, lo cual no representa la totalidad de la confor- 
mación de las familias de niños y adolescentes estadounidenses en México, como se muestra en el Cuadro 4.

\section{Cuadro 3}

Porcentaje de población de 6 a 17 años nacida en Estados Unidos y que reside en México, según tamaño del lugar de residencia y región de llegada; 1990-2015

\begin{tabular}{lllll}
\hline Tamaño lugar residencia & $\mathbf{1 9 9 0}$ & $\mathbf{2 0 0 0}$ & $\mathbf{2 0 1 0}$ & $\mathbf{2 0 1 5}$ \\
\hline Rural (menos de 2 500 habs.) & 21.1 & 19.1 & 23.9 & 25.1 \\
Urbano (más de 2 500 habs.) & 78.9 & 80.9 & 76.1 & 74.9 \\
\hline Región de llegada & & & & \\
Norte & 50.5 & 50.4 & 45.2 & 42.3 \\
Tradicional & 41.3 & 35.3 & 31.7 & 31.7 \\
Centro & 5.9 & 9.8 & 14.5 & 15.8 \\
Sur-sureste & 2.3 & 4.4 & 8.7 & 10.2 \\
\hline
\end{tabular}

Fuente: Editado de Zúñiga y Giorguli, 2019.

\section{Cuadro 4}

Porcentaje de población de 6 a 17 años nacida en Estados Unidos que reside en México, con corresidencia compartida, según grupos de edad; 2015

\begin{tabular}{llc}
\hline Edad & Corresidencia con padres & $\begin{array}{c}\text { Nacidos } \\
\text { en Estados Unidos }\end{array}$ \\
\hline De 6 a 11 años & Con ambos padres & $66.1 \%$ \\
& Sólo con la madre & $24.8 \%$ \\
& Sólo con el padre & $2.4 \%$ \\
& Con ninguno & $6.6 \%$ \\
\hline De 12 a 15 años & Con ambos padres & $62.6 \%$ \\
& Sólo con la madre & $25.6 \%$ \\
& Sólo con el padre & $3.6 \%$ \\
& Con ninguno & $8.2 \%$ \\
\hline De 16 a 17 años & Con ambos padres & $58.3 \%$ \\
& Sólo con la madre & $23.9 \%$ \\
& Sólo con el padre & $4.0 \%$ \\
& Con ninguno & $13.8 \%$
\end{tabular}

Fuente: Editado de Zúñiga y Giorguli, 2019. 


\section{Los mexicanos que se educaron en Estados Unidos}

A diferencia del grupo nacido en Estados Unidos, los mexicanos retornados a México conforman un frente de más de 60 mil menores de edad. Esto comprueba que los mexicanos de primera generación que llegaron a Estados Unidos conformaron familias prósperas que ahora se encuentran en México. Otra diferencia con respecto a la población anterior es que los mexicanos retornados se distinguen por ser un grupo con mayor exposición escolar en Estados Unidos; por ejemplo, el grupo principal es el de 10 a 14 años, los cuales están en edad escolar para estudiar el final de la educación primaria y los tres años de la secundaria.

\section{Gráfica 5}

Población de 5 a 17 años nacida en México con residencia en Estados Unidos en el quinquenio anterior, según grupos de edad; 2000 y 2010

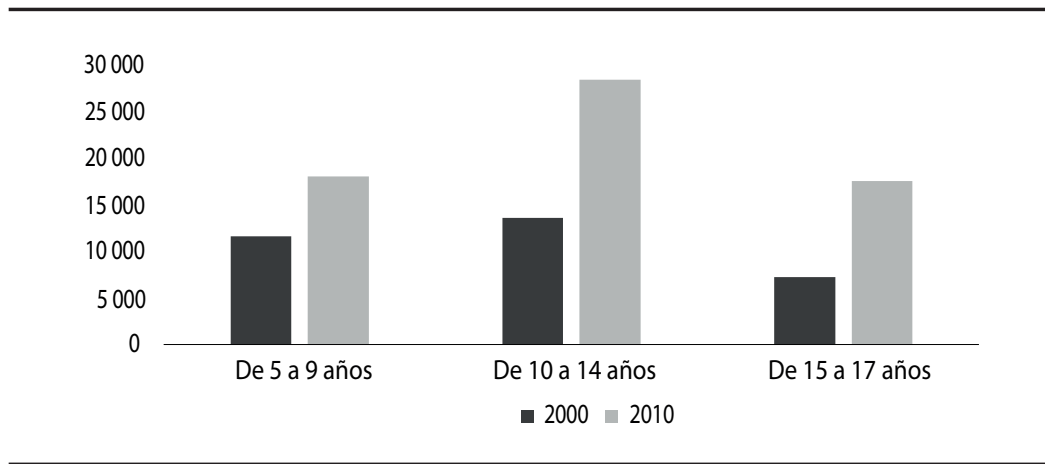

Fuente: Elaboración propia con información del Censo de Población y Vivienda INEGI, 2000 y 2010 (INEGI, 2017).

Con rasgos similares a los grupos de menores nacidos en Estados Unidos, la población de origen mexicano tiende a migrar más constantemente hacia localidades rurales, manteniendo un patrón más generalizado de dispersión territorial, aun cuando, en términos absolutos, la llegada es principalmente a destinos urbanos. Considerando que los estados de California, Texas, Illinois y Arizona concentran la mayor cantidad de población mexicana en grandes áreas metropolitanas y que son precisamente de esas zonas las mayores olas de expulsión de mexicanos hacia México (Stepler y Lopez, 2016), se hacen más evidentes las rupturas y discontinuidades cotidianas de estos niños y adolescentes al llegar al país (Coe, Reynolds, Boehm, Hess y Rae-Espinoza, 2011): después de todo, no es lo 
mismo educarse en una ciudad como Los Ángeles, a hacerlo en un pueblo cañaveral de Morelos.

En este sentido, los grupos nacidos en México tienden a dispersarse más equitativamente en el territorio mexicano, considerando que después de la región Tradicional, las que concentran mayor cantidad de lugares de origen de migrantes de retorno son la Norte y la Centro. Éste es el caso de Beto, quien, después de vivir más de 14 años en Santa Ana, California, reingresó a México para vivir en comunidades rurales y agrícolas de Morelos y del Estado de México.

\section{Cuadro 5}

Porcentaje de población de 6 a 17 años nacida en México con residencia en Estados Unidos en el quinquenio anterior, según tamaño del lugar de residencia y región de llegada; 1990-2015

\begin{tabular}{lllll}
\hline Tamaño lugar residencia & $\mathbf{1 9 9 0}$ & $\mathbf{2 0 0 0}$ & $\mathbf{2 0 1 0}$ & $\mathbf{2 0 1 5}$ \\
\hline Rural (menos de 2 500 habs.) & 17.7 & 21.5 & 23.8 & 24.4 \\
Urbano (más de 2500 habs.) & 82.3 & 78.5 & 76.2 & 75.6 \\
\hline Región de llegada & & & & \\
Norte & 30.6 & 28.7 & 30.4 & 25.2 \\
Tradicional & 54.1 & 47.9 & 36.1 & 36.9 \\
Centro & 11.9 & 15.7 & 17.8 & 24.2 \\
Sur-sureste & 3.4 & 7.7 & 15.8 & 13.7 \\
\hline
\end{tabular}

Fuente: Editado de Zúñiga y Giorguli, 2019.

Si bien la corresidencia compartida con ambos padres sigue mostrando mayor predominancia estadística, sigue siendo significativa la cantidad de niños y adolescentes retornados que se encuentran separados de su padre. Por ejemplo, Marco está separado físicamente de su padre porque vive con su mamá en Morelos, mientras que su papá vive en Chicago porque tiene documentos para trabajar legalmente. Pero a diferencia de los niños nacidos en Estados Unidos, Marco y Beto están “enjaulados" en México porque no tienen la ciudadanía estadounidense, lo que obstaculiza su tránsito entre ambos países, aun cuando ellos imaginan constantemente estar en Estados Unidos con sus amigos, con el resto de su familia, estudiando o ganando dinero para enviar recursos a sus padres o pedirlos para que la familia pueda reunificarse en el norte (Zúñiga y Giorguli, 2019). 


\section{Cuadro 6}

Porcentaje de población de 6 a 17 años nacida en México y con residencia en Estados Unidos en el quinquenio anterior, según corresidencia en México y grupos de edad; 2015

\begin{tabular}{llc}
\hline Edad & $\begin{array}{c}\text { Corresidencia } \\
\text { con los padres }\end{array}$ & $\begin{array}{c}\text { Nacidos } \\
\text { en Estados Unidos }\end{array}$ \\
\hline De 6 a 11 años & Con ambos padres & $71.0 \%$ \\
& Sólo con la madre & $21.1 \%$ \\
& Sólo con el padre & $1.9 \%$ \\
& Con ninguno & $6.0 \%$ \\
\hline De 12 a 15 años & Con ambos padres & $63.9 \%$ \\
& Sólo con la madre & $24.9 \%$ \\
& Sólo con el padre & $3.0 \%$ \\
& Con ninguno & $8.2 \%$ \\
\hline De 16 a 17 años & Con ambos padres & $50.6 \%$ \\
& Sólo con la madre & $26.4 \%$ \\
& Sólo con el padre & $4.8 \%$ \\
& Con ninguno & $18.3 \%$ \\
\hline
\end{tabular}

Fuente: Editado de Zúñiga y Giorguli, 2019.

\section{Niños y adolescentes que retornan a Estados Unidos}

Sin lugar a dudas, el estado legal, el lugar de nacimiento y las amplias redes familiares son determinantes para facilitar o entorpecer la movilidad internacional de los niños y adolescentes migrantes. Aquellos que nacieron en Estados Unidos, como María, Lulú y Flor, pueden retornar "sencillamente" al país en el que nacieron porque su pasaporte lo permite. Sin embargo, el caso de las hermanas es distinto porque la unidad familiar fue la causa principal del retorno, y la separación de algún miembro -aun cuando tienen familia extendida en San Diego- no es un motivo suficiente para que ellas tomen la decisión de migrar en un momento cercano. Por otro lado, Flor, al tener dos fuertes vínculos emocionales y familiares -su padre en Portland y su madre en el Estado de México-, tiene la opción de transitar más libremente entre los dos países.

A diferencia de las primeras cuatro agrupaciones, el perfil en el que se encuentra Flor es el de un patrón de movilidad que es bastante reciente como para tener información estadística que reporte el U.S. Census Bureau. 
Hasta el momento, el American Community Census no permite capturar las entradas internacionales de menores nacidos en Estados Unidos, así como tampoco identifica los estados, ciudades o distritos escolares a los que retornan estos niños y adolescentes que se han escolarizado en dos sistemas educativos diferentes. Más aún, se desconocen las trayectorias migratorias de niñas y niños como María, que inician su socialización escolar en México y que fácilmente podrían transitar entre ambos países.

\section{Conclusiones}

Nos encontramos frente a nuevas experiencias migratorias que trastocan las estructuras demográficas de México y Estados Unidos, así como plantean nuevos retos analíticos, metodológicos y conceptuales para la comprensión de las experiencias subjetivas de los niños y adolescentes que experimentan estas nuevas trayectorias transnacionales. México ha dejado de ser el principal país expulsor de migrantes hacia Estados Unidos y se ha convertido en un país receptor de mexicanos socializados en Estados Unidos, así como de estadounidenses socializados escolarmente como miembros de la sociedad de aquel país y socializados como integrantes de familias transnacionales.

Estos menores experimentan negociaciones personales sobre normas, interpretaciones, comportamientos y saberes que han aprendido en uno o en varios sistemas escolares, que y los hace pertenecer a una nueva generación de migrantes que tiene en su futuro, por un lado, la libertad de afiliarse culturalmente en geografías transnacionales y, por otro, la dificultad de no poder continuar su carrera escolar y laboral simultáneamente en Estados Unidos y México. Los niños y adolescentes que conforman la generación 0.5 se caracterizan por agruparse de cinco maneras distintas dependiendo de: el país de nacimiento, el inicio de su vida escolar, la interrupción y continuación de la vida escolar en otro país y el posible retorno y circulación entre distintos Estados-nación.

En México, estos menores muestran patrones nuevos de socialización, como el caso de los niños que Rumbaut $(1997 ; 2004)$ identifica como los de la infancia temprana y la media, a diferencia de los más de 60 mil mexicanos que están regresando al país, pero que se caracterizan por ser la población con mayor antigüedad en Estados Unidos. En ambos casos, los lugares tradicionales de recepción en México se están diluyendo para abrirle paso a una dispersión más generalizada, a la llegada de la generación 0.5 .

En síntesis, esta nueva identificación de grupos, tipologías y perfiles de menores migrantes internacionales requiere de la realización de investiga- 
ciones cualitativas que busquen acompañar longitudinalmente a los niños durante su vida. Con esto, se tendrá una comprensión más fina y auténtica de la infancia en tiempos de importantes cambios en los modelos de globalización mundial.

\section{Bibliografía}

Berger, P. y Luckmann, T. (2003). La construcción social de la realidad. Argentina: Amorrortu.

Coe, C., Reynolds, R., Boehm, D. A., Hess, J. M. y Rae-Espinoza, H. (2011). Everyday ruptures: Children, youth, and migration in global perspective. Nashville: Vanderbilt University Press.

Conapo. (2011). Índices de intensidad migratoria México-Estados Unidos 2010. Ciudad de México: Consejo Nacional de Población. Recuperado de http://www.conapo.gob.mx/swb/conapo(Indices_de_intesidad_ migratoria_Mexico-Estados_Unidos_2010

Dobson, M. (2009). Unpacking children in migration research. Journal Children's Geographies, 7(3), 355-360. Recuperado de https://www. tandfonline.com/doi/abs/10.1080/14733280903024514

Dubet, F. (2002). El declive de la institución. Profesiones, sujetos e individuos ante la reforma del Estado. Barcelona: Gedisa.

Durkheim, É. (1976). Educación como socialización. Salamanca: Sígueme. Escobar, A., Lowell, L. y Martin, S. (2013). Binational dialogue on Mexican migrants in the U.S. and in Mexico. México: CIESAS / Universidad de Georgetown.

Gaitán, L. (2006). La nueva sociología de la infancia. Aportaciones de una mirada distinta. Política y Sociedad, 43(1), 9-26. Recuperado de https://revistas.ucm.es/index.php/POSO/article/view/23767

Giorguli, S. y Gutiérrez, E. (2011). Niños y jóvenes en el contexto en la migración internacional entre México y Estados Unidos. Coyuntura Demográfica, 1, 21-25. Recuperado de http://coyunturademografica.somede. org/wp-content/plugins/coyuntura_demografica/DEMOGRAFICA/ ARTICULOS/PUB-2011-01-005.pdf

Gonzales, R. G. (2010). On the wrong side of the tracks: Understanding the effects of school structure and social capital in the educational pursuits of undocumented immigrant students. Peabody Journal of Education, 85(4), 469-485. Recuperado de https://www.tandfonline.com/doi/abs/1 0.1080/0161956X.2010.518039 
Gonzalez-Barrera, A. (2015). More Mexicans leaving than coming to the U.S. Washington, DC: Pew Hispanic Center. Recuperado de https:// www.pewhispanic.org/2015/11/19/more-mexicans-leaving-thancoming-to-the-u-s/

Hamann, E. T., Zúñiga, V. y Sánchez, J. (2006). Pensando en Cynthia y en su hermana: Educational implications of United States-Mexico transnationalism for children. Journal of Latinos and Education, 5(4), 253274. Recuperado de https://www.tandfonline.com/doi/abs/10.1207/ s1532771xjle0504_3

Hatfield, M. E. (2010). Children moving 'home'? Everyday experiences of return migration in highly skilled households. Childhood, 17(2), 243-257. Recuperado de https://journals.sagepub.com/doi/ pdf $/ 10.1177 / 0907568210365747$

INEGI. (2017). Serie histórica censal e intercensal (1990-2010). Aguascalientes: Instituto Nacional de Estadística y Geografía. Recuperado de http://www.beta.inegi.org.mx/proyectos/ccpv/cpvsh/ default.html

López, G. y Radford, J. (2017). Statistical portrait of the foreign-born population in the United States, 2015. Washington, DC: Pew Hispanic Center.

Orellana, M. F., Thorne, B., Chee, A. y Lam, E. (2001). Transnational chilhoods: The participation of children in processes of family migration. Social Problems, 48(4), 572-591. Recuperado de https://www.sesp. northwestern.edu/docs/publications/3445846804bd0e836e0552.pdf

Panait, C. y Zúñiga, V. (2016). Children circulating between the U.S. and Mexico: Fractured schooling and linguistic ruptures. Mexican Studies / Estudios Mexicanos, 32(2), 226-251. Recuperado de https://msem. ucpress.edu/content $/ 32 / 2 / 226$

Passel, J. S. y Cohn, D. (2009). Mexican immigrants: How many come? How many leave? Washington, DC: Pew Hispanic Center. Recuperado de https://www.pewhispanic.org/2009/07/22/mexican-immigrantshow-many-come-how-many-leave/

Passel, J. S. y Cohn, D. (2016). Size of U.S. unauthorized immigrant workforce stable after the Great Recession. Washington, DC: Pew Hispanic Center. Recuperado de https://www.pewhispanic.org/2016/11/ 03/size-of-u-s-unauthorized-immigrant-workforce-stable-after-thegreat-recession/

Passel, J. S., Cohn, D. y Gonzalez-Barrera, A. (2012), Net migration from Mexico falls to zero-and perhaps less. Washington, DC: Pew Hispanic Center. Recuperado de https://www.pewhispanic.org/2012/04/23/ net-migration-from-mexico-falls-to-zero-and-perhaps-less/ 
Patten, E. (2016). The nation's Latino population is defined by its youth. Washington, DC: Pew Hispanic Center. Recuperado de https://www. pewhispanic.org/2016/04/20/the-nations-latino-population-is-definedby-its-youth/

Portes, A. y Hao, L. (2004). The schooling of children of immigrants: Contextual effects on the educational attainment of the second generation. Proceedings of the National Academy of Sciences of the United States of America, 101(33), 11920-11927. Recuperado de https://www.pnas. org/content $/ 101 / 33 / 11920$

Prout, A. (2011). Taking a step away from modernity: Reconsidering the new sociology of childhood. Global Studies of Childhood, 1(1), 4-14. Recuperado de https://journals.sagepub.com/doi/10.2304/gsch.2011.1.1.4

Ramírez, T. y Meza, L. (2011). Emigración México-Estados Unidos: balance antes y después de la recesión económica estadounidense. En Conapo (ed.), La situación demográfica de México (pp. 241-259). Ciudad de México: Consejo Nacional de Población.

Román, B. (2017). “Pa'cuando me regrese, can we speak in English?”: Trayectorias de menores migrantes que llegan a México. (Tesis de doctorado en Ciencias Sociales, México, Instituto Tecnológico y de Estudios Superiores de Monterrey). Recuperado de https://www.isa-sociology. org/en/junior-sociologists/dissertation-abstracts/list-of-abstracts/1101

Román, B. y Carrillo, E. (2017). Bienvenido a la escuela: experiencias escolares de alumnos transnacionales de Morelos, México. Sinéctica, Revista Electrónica de Educación, 48, 1-19. Recuperado de https://www. redalyc.org/jatsRepo/998/99856084009/html/index.html

Román, B., Carrillo, E. y Hernández-León, R. (2016). Moving to the 'homeland': Children's narratives of migration from the United States to Mexico. Mexican Studies / Estudios Mexicanos, 32(2), 252-275. Recuperado de https://msem.ucpress.edu/content/32/2/252

Román, B. y Zúñiga, V. (2014). Children returning from the U.S. to Mexico: School sweet school? Migraciones Internacionales, 7(4), 277-286. Recuperado de http://www.scielo.org.mx/pdf/migra/v7n4/v7n4a10.pdf

Rumbaut, R. (1997). Ties that bind: Immigration and immigrant families in the United States. En A. Booth, A. C. Crouter y N. Landale (coords.), Immigration and the family. Research and policy on U.S. immigrants (pp. 3-61). Nueva Jersey: Lawrence Erlbaum.

Rumbaut, R. (2004). Ages, life stages, and generational cohorts: Decomposing the immigrant first and second generations in the United States. The Internacional Migration Review, 38(3), 1160-1205. Recuperado 
de https://www.jstor.org/stable/27645429?seq=1\#metadata_info_tab_ contents

Sánchez, J. y Zúñiga, V. (2010). Trayectorias de los alumnos transnacionales en México. Propuesta intercultural de atención educativa. Trayectorias, 12(30), 5-23. Recuperado de http://www.redalyc.org/articulo. oa? id $=60713488002$

Stepler, R. y Lopez, M. H. (2016). U.S. Latino population growth and dispersion has slowed since onset of the Great Recession. Washington, DC: Pew Hispanic Center. Recuperado de https://www.pewhispanic. org/2016/09/08/latino-population-growth-and-dispersion-has-slowedsince-the-onset-of-the-great-recession/

U.S. Census Bureau (2010), 2010 American Community Survey 1-Year Estimates. Recuperado de https://www.census.gov/programs-surveys/acs/ data.html

U.S. Census Bureau (2016), 2016 American Community Survey 1-Year Estimates. Recuperado de https://www.census.gov/programs-surveys/acs/ data.html

Valdés, G. (1996). Con respeto: Bridging the distances between culturally diverse families and schools. An ethnographic portrait. Nueva York: Teachers College Press.

Zhou, M. (1997). Segmented assimilation: Issues, controversies, and recent research on the new second generation. International Migration Review, 31(4), 975-1008. Recuperado de https://www.researchgate.net/ publication/11129264_Segmented_Assimilation_Issues_Controversies and_Recent_Research_on_the_New_Second_Generation

Zúñiga, V. (2011). La escuela incluyente y justa. Antología comentada al servicio de los maestros de México. México: Fondo Editorial de Nuevo León / Universidad de Monterrey.

Zúñiga, V. (2013). Migrantes internacionales en las escuelas mexicanas: desafíos actuales y futuros de política educativa. Sinéctica. Revista Electrónica de Educación, 40, 1-12. Recuperado de https://sinectica.iteso. $\mathrm{mx} /$ index.php/SINECTICA/article/view/50

Zúñiga, V. (2015). Niños y adolescentes separados de sus familias por la migración internacional: el caso de cuatro estados de México. Estudios Sociológicos, 97, 145-168. Recuperado de http://www.scielo.org.mx/ pdf/es/v33n97/2448-6442-es-33-97-00145.pdf

Zúñiga, V. y Giorguli, S. (2019). Niñas y niños migrantes internacionales en México: la generación 0.5. Ciudad de México: El Colegio de México, A.C. 
Zúñiga, V. y Hamann, E. T. (2011). Volviendo a visitar la noción de transnacionalidad: comunicación transfronteriza y redes diaspóricas en alumnos migrantes internacionales en las escuelas de México. En J. J. Olvera. y B. D. Vázquez (coords.), Procesos comunicativos en la migración: de la escuela a la feria popular (pp. 29-59). México: El Colegio de la Frontera Norte / Universidad de Monterrey / Universidad Regiomontana / Instituto Tecnológico y de Estudios Superiores de Monterrey.

Zúñiga, V. y Hamann, E. T. (2014). Going to a home you have never been to: The return migration of Mexican and American-Mexican children. Journal Children's Geographies, 13(6), 643-655. Recuperado de https://www.tandfonline.com/doi/abs/10.1080/14733285.2014.936364 Zúñiga, V., Hamann, E. T. y Sánchez, J. (2008). Alumnos transnacionales, escuelas mexicanas frente a la globalización. Ciudad de México: Secretaría de Educación Pública.

Zúñiga, V. y Hernández-León, R. (2005). New destinations: Mexican immigration in the United States. Nueva York: Russell Sage Foundation. Zúñiga, V. y Vivas, M. (2014). Divided families, fractured schooling in Mexico: Educational consequences of children exposition to international migration. Cahiers CEMCA, 6, 3-18. Recuperado de https://www. researchgate.net/publication/289378950_Divided_families_fractured_ schooling_in_Mexico_educational_consequences_of_children_ exposition_to_international_migration

\section{Acerca de los autores}

Eduardo Carrillo Cantú es doctorante en Ciencias Sociales por el Tecnológico de Monterrey, con una investigación que explora y analiza las formas de exclusión e inclusión escolar, en escuelas de educación básica de México, de alumnos transnacionales que migran de Estados Unidos a este país. Es sociólogo por la Universidad de Monterrey, en donde fue becario Conacyt nivel I. Su línea de investigación versa sobre las narrativas de retorno de menores migrantes internacionales en México, centrándose en la perspectiva de los menores y de sus tutores para conocer la rupturas pedagógicas, lingüísticas y culturales que ocurren fuera y dentro del aula en ambos países. Ha publicado en las revistas Mexican Studies / Estudios Mexicanos y Sinéctica. Actualmente colabora en el proyecto "Migración internacional y exclusión escolar: hacia la construcción de políticas de bienvenida para los menores que retornan de Estados Unidos a México" (Conacyt 2016-011972). ORCID: https://orcid.org/0000-0003-3264-265X 
Betsabé Román González es catedrática Conacyt en El Colegio de Sonora, donde dirige el proyecto "Tránsito y retorno de la niñez migrante: características y política pública en Sonora". Cuenta con un postdoctorado en Educación Transnacional del Centro USMEX de la Universidad de California, San Diego. Se doctoró en Ciencias Sociales por el Tecnológico de Monterrey, y tiene una especialidad en Antropología Social por el CIESAS Noreste. Desde 2011 dirige el único estudio longitudinal y multisituado con niños, niñas y adolescentes (NNA) migrantes que retornan a México y a Estados Unidos. A partir de 2018 colabora en los estudios y actividades del Programa de Estudios Transfronterizos y el Seminario Niñez Migrante de El Colegio de Sonora, en las líneas de migración de retorno y de tránsito de NNA migrantes en ese estado. A partir de estas experiencias ha diseñado e impartido talleres de concientización sobre temáticas migratorias y de capacitación sobre inclusión pedagógica de NNA migrantes a docentes de educación básica en diferentes estados de México; además, colabora en la creación de políticas públicas y educativas desde y para los NNA migrantes en el país. Actualmente participa en el proyecto "Migración internacional y exclusión escolar: hacia la construcción de políticas de bienvenida para los menores que retornan de Estados Unidos a México" (Conacyt 2016-011972). ORCID: https://orcid.org/0000-0001-9566-3533

Recepción: 31 de diciembre de 2017. Aceptación: 15 de mayo de 2019. 
